\title{
Concept of a tunable source of coherent THz radiation driven by a plasma modulated electron beam
}

Cite as: Phys. Plasmas 25, 043111 (2018); https://doi.org/10.1063/1.5017551

Submitted: 28 November 2017 . Accepted: 02 April 2018 . Published Online: 17 April 2018

H. Zhang, I. V. Konoplev (D, G. Doucas, and J. Smith
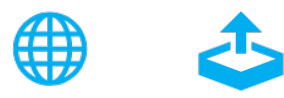

View Online

\section{ARTICLES YOU MAY BE INTERESTED IN}

Observation of coherent Smith-Purcell and transition radiation driven by single bunch and micro-bunched electron beams

Applied Physics Letters 112, 053501 (2018); https://doi.org/10.1063/1.5009396

Terahertz radiation generation by beating of two laser beams in a collisional plasma with oblique magnetic field

Physics of Plasmas 25, 023514 (2018); https://doi.org/10.1063/1.5005918

Plasma physics and related challenges of millimeter-wave-to-terahertz and high power microwave generation

Physics of Plasmas 15, 055502 (2008); https://doi.org/10.1063/1.2838240

Where in the world is AIP Publishing?

Find out where we are exhibiting next 


\title{
Concept of a tunable source of coherent THz radiation driven by a plasma modulated electron beam
}

\author{
H. Zhang, ${ }^{1, a)}$ I. V. Konoplev, ${ }^{1}$ G. Doucas, ${ }^{1}$ and J. Smith ${ }^{2}$ \\ ${ }^{1}$ John Adams Institute for Accelerator Science, University of Oxford, Denys Wilkinson Building, Keble Road, \\ Oxford OX1 3RH, United Kingdom \\ ${ }^{2}$ Tech-X UK Ltd., The Innovation Centre, Sci-Tech Daresbury, Daresbury WA4 4FS, United Kingdom
}

(Received 28 November 2017; accepted 2 April 2018; published online 17 April 2018)

\begin{abstract}
We have carried out numerical studies which consider the modulation of a picosecond long relativistic electron beam in a plasma channel and the generation of a micro-bunched train. The subsequent propagation of the micro-bunched beam in the vacuum area was also investigated. The same numerical model was then used to simulate the radiation arising from the interaction of the microbunched beam with a metallic grating. The dependence of the radiation spectrum on the parameters of the micro-bunched beam has been studied and the tunability of the radiation by the variation of the micro-bunch spacing has been demonstrated. The micro-bunch spacing can be changed easily by altering the plasma density without changing the beam energy or current. Using the results of these studies, we develop a conceptual design of a tunable source of coherent terahertz $(\mathrm{THz})$ radiation driven by a plasma modulated beam. Such a source would be a potential and useful alternative to conventional vacuum $\mathrm{THz}$ tubes and $\mathrm{THz}$ free-electron laser sources. Published by AIP Publishing. https://doi.org/10.1063/1.5017551
\end{abstract}

\section{INTRODUCTION}

The terahertz $(\mathrm{THz})$ spectral region covers the frequency range from 0.1 to $10 \mathrm{THz}$. This is a range of interest to many areas, including information and communication technology, biology, medical sciences, non-destructive detection, and security. ${ }^{1-3}$ Due to the development of accelerator and femtosecond (fs) laser technologies around the world, linear accelerators (LINACs) have become more compact and affordable, and relativistic femtosecond electron beams with single particle energy in the region $10 \mathrm{MeV}$ to $50 \mathrm{MeV}$ are readily available. It has been suggested that $\mathrm{THz}$ radiation sources could be developed which are driven by accelerators in this energy range. ${ }^{4-9}$ The attractive features of such sources include tunability over a broad frequency and power range, from $0.1 \mathrm{THz}$ to $10 \mathrm{THz}$ and from $10 \mathrm{~W}$ to $10 \mathrm{MW}$ (pulse regime), respectively.

Coherent radiation generated by a short (in comparison with operating wavelength) relativistic electron bunch (a conventional method used in many laboratories ${ }^{10-13}$ ) has a broadband spectrum, while the conversion of the kinetic energy of the beam into $\mathrm{THz}$ radiation is not very efficient. Due to strong space-charge forces, it is difficult to compress a high charge $(>1 \mathrm{nC})$ into a very short bunch, especially when the beam has a relatively low energy $(<10 \mathrm{MeV})$. This limits the number of electrons in the bunch and thus the peak power generated. To solve this issue, a pre-bunched electron beam consisting of a train of micro-bunches can be used; this would maintain the total high charge within the micro-bunch train and the coherence of the output radiation. Reducing the space-charge effect also helps to overcome other technical issues such as RF breakdown and overheating.

There are a number of methods for generating trains of fs-long micro-bunches with bunch spacing of picoseconds

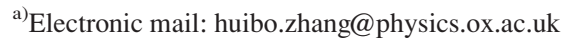

and sub-picoseconds. The first is to modulate the electron beam by using laser pulses, either by direct modulation of the emission at the photocathode ${ }^{8,14-16}$ or by laser-beam interaction inside an undulator in the beamline. ${ }^{17,18}$ These methods have been proved efficient in bunching electron beams; however, the introduction of lasers along the beamline makes the whole system more complex and expensive. Other methods include the transformation of the beam energy modulation into a density modulation ${ }^{7,19}$ and the beam slicing technique, ${ }^{20-22}$ together with the utilization of appropriate beam optics. The last two methods require no additional complex components, while the tunability is limited due to the constraints of the design. It has been proved that by importing a long electron beam into a plasma channel, the beam can be divided into a train of micro-bunches via the self-modulation instability (SMI) with the spacing defined by the plasma density. ${ }^{23,24}$ The self-modulation is due to the self-excited wakefield in the transverse plane. The biggest advantage of this approach compared with the others listed is its simplicity, as the micro-bunch spacing can be tuned by changing the gas pressure in the plasma capillary (assuming the plasma is produced by the voltage discharge in gas) and thus the plasma density.

In this paper, we discuss the steps required to observe tunable $\mathrm{THz}$ radiation from a picosecond long relativistic electron beam using the plasma modulation technique. We start with the basic principles of the beam modulation in the plasma, demonstrating efficient pre-bunching of the beam and formulating the challenges associated with the utilization of such a beam. The influence of different parameters on the beam modulation is investigated in Secs. II and III. The injection and propagation of the modulated beam into vacuum area are investigated numerically using the Particle-inCell (PIC) code VSim in Sec. IV. This section also considers 
the conditions under which the beam modulation is preserved in the vacuum area for a distance long enough (in our case $10 \mathrm{~cm}$ ) to observe coherent radiation. Section V discusses the generation of coherent radiation by the interaction of a micro-bunched beam with a periodic metal grating. The conclusions are presented in Sec. VI.

\section{SELF-MODULATION INSTABILITY AND BEAM MODULATION IN PLASMA-LINEAR THEORY}

A plasma wakefield can be generated by an electron beam moving through a plasma channel. To evaluate the radial forces acting upon the beam and the beam dynamics, the slow amplitude envelope equation for the azimuthally symmetric beam can be used ${ }^{25-27}$ (see Appendix A for a more detailed discussion). This treatment uses a reference frame moving with the beam and the beam is divided into thin (as compared with plasma wavelength) slices; all electrons inside a slice have the same longitudinal velocity meaning that a particle inside such a slice can only move within the transverse plane. This model is reasonable and valid for a relatively small energy modulation of a relativistic $(>10 \mathrm{MeV})$ electron beam and the longitudinal modulation of the velocities of the particles inside the slice can be neglected. A typical set of beam parameters used in our simulations are listed in Table I.

The initial beam transverse profile is assumed to be Gaussian with the transverse size $\sigma_{r}$ changing with the propagation distance in the plasma, while the longitudinal profile can be of an arbitrary geometry. In particular, we are interested in the trapezoidal longitudinal profile, meaning that the beam has a uniform density in the central section, as well as rise and decay slopes (with the corresponding rise/decay times), which are defined by the time from zero to maximum density. If the rise and decay times are equal to zero, the longitudinal profile is square (bunch with a square profile has been demonstrated in Refs. 20 and 21). The density distribution $n_{b}$ of a square beam with the parameters listed in Table I can be defined as $n_{b}(r)=n_{b 0} \exp \left(-r^{2} / 2 \sigma_{r}^{2}\right)$ and $n_{b 0}=Q /$ $2 \pi e L \sigma_{r 0}^{2}=2.3 \times 10^{13} / \mathrm{cm}^{3}$. Assuming that the plasma wavelength $\lambda_{p}$ is $300 \mu \mathrm{m}(1 \mathrm{THz})$, the plasma density $n_{p}$ can be calculated to be $1.24 \times 10^{16} / \mathrm{cm}^{3}$ (plasma frequency $\omega_{p}$ $\left.=\sqrt{n_{p} e^{2} / m_{0} \epsilon_{0}}\right)$, and $n_{b 0} / n_{p} \approx 0.2 \%$, meaning that one can consider the beam-plasma interaction is in the linear regime.

There are a number of parameters which can be varied to maximise the beam modulation. Considering the beam parameters listed in Table I and using the beam envelope model

TABLE I. Beam and plasma parameters.

\begin{tabular}{lcc}
\hline \hline Description & Parameters & Value \\
\hline Beam energy & $E$ & $50 \mathrm{MeV}$ \\
Beam charge & $Q$ & $1 \mathrm{nC}$ \\
Beam length & $L$ & $3000 \mu \mathrm{m}$ \\
Beam transverse size & $\sigma_{r 0}$ & $120 \mu \mathrm{m}$ \\
Beam emittance & $\varepsilon$ & $1 \mathrm{~mm} \mathrm{mrad}$ \\
Beam rise profile & $\ldots$ & $\mathrm{Linear}_{2}$ sinusoidal \\
Beam rise time & $\delta$ & $0,0.5 T_{p}, T_{p}, 1.5 T_{p}$ \\
Plasma wavelength & $\lambda_{p}$ & $150 \mu \mathrm{m}, 200 \mu \mathrm{m}, 300 \mu \mathrm{m}$ \\
\hline \hline
\end{tabular}

described above, the evolution of the bunch transverse size $\sigma_{r}(z, \xi)$ as a function of the longitudinal propagation distance $z$ and the relative coordinate of the beam $\xi$ (in a reference frame moving with the beam) can be studied. The results of the semianalytical studies of the beam modulation for different plasma densities are shown in Fig. 1(a). It shows that a long bunch with a length of 10 ps has been modulated into a micro-bunch train, with the periodicity defined by the plasma frequency after propagating through $3.6 \mathrm{~cm}$ in the plasma channel. The micro-bunches are equidistant, with separations of $300 \mu \mathrm{m}$, $200 \mu \mathrm{m}$ and $150 \mu \mathrm{m}$ corresponding to a specific plasma wavelength defined by the plasma density. For a given length $L_{p}$ of the plasma channel, increasing the plasma density results in an increase in the modulation frequency and a decrease in the amplitude of the modulations (smaller transverse focusing and defocusing forces acting upon the beam). As far as the evolution of the beam modulation is concerned [Fig. 1(b)], we see that a longer propagation distance $L_{p}$ results in a higher beam modulation amplitude for fixed beam parameters. An initially unmodulated beam after a short propagation distance $\left(<15 \lambda_{p}\right)$ (solid green line) has no obvious modulation. With the increase in the propagation distance, a periodic modulation occurs along the beam which can clearly be seen in the range from $45 \lambda_{p}$ to $90 \lambda_{p}$. At longer propagation distances $\left(>90 \lambda_{p}\right)$, the amplitude of the modulation starts to vary. The modulation is stronger at the tail and weaker at the head (non-uniform modulation). The interaction between the beam and the wakefield becomes non-linear and more accurate numerical modelling is required to describe the beam-plasma interaction.

To further improve the beam modulation in a plasma channel, the initial bunch profile must also be considered. Previous studies ${ }^{28}$ have shown that the beam modulation depends on the longitudinal rise time and the strong self-modulation instability is excited from a sharp rise profile, with a general rule that a shorter rise time leads to a larger modulation rate. Nevertheless, this instability can also be observed for electron beams with a relatively long rise time which simplifies the beam profiling. The dependence of the beam modulation on the initial rise profile is shown in Figs. 1(c) and 1(d). In particular, two initial longitudinal rise profiles were considered: linear and sinusoidal. In the case of the linear profile, if the rise time is equal to a multiple of plasma period $\left(\delta=n T_{p}\right)$, only beam compression (focusing) takes place; if the rise profile is not linear, some weak periodical modulations are still visible even if the rise time $\delta=n T_{p}$. (further detailed analysis can be found in Appendix B).

There are some limitations of the above analytical model as it is based on the assumption of a linear interaction between the beam and the plasma. As a result, this model is only applicable to the description of small perturbations (at the start of the interaction). Nevertheless, it helps limit the range of the parameters used at the next stage of the investigations, which uses numerical models.

\section{BEAM MODULATION IN PLASMA-NUMERICAL STUDIES}

VSim is a full 3D PIC software, ${ }^{29}$ and it is capable of parallel processing which enables the computationally intensive, self-consistent modelling of the plasma-beam interaction. ${ }^{29}$ 

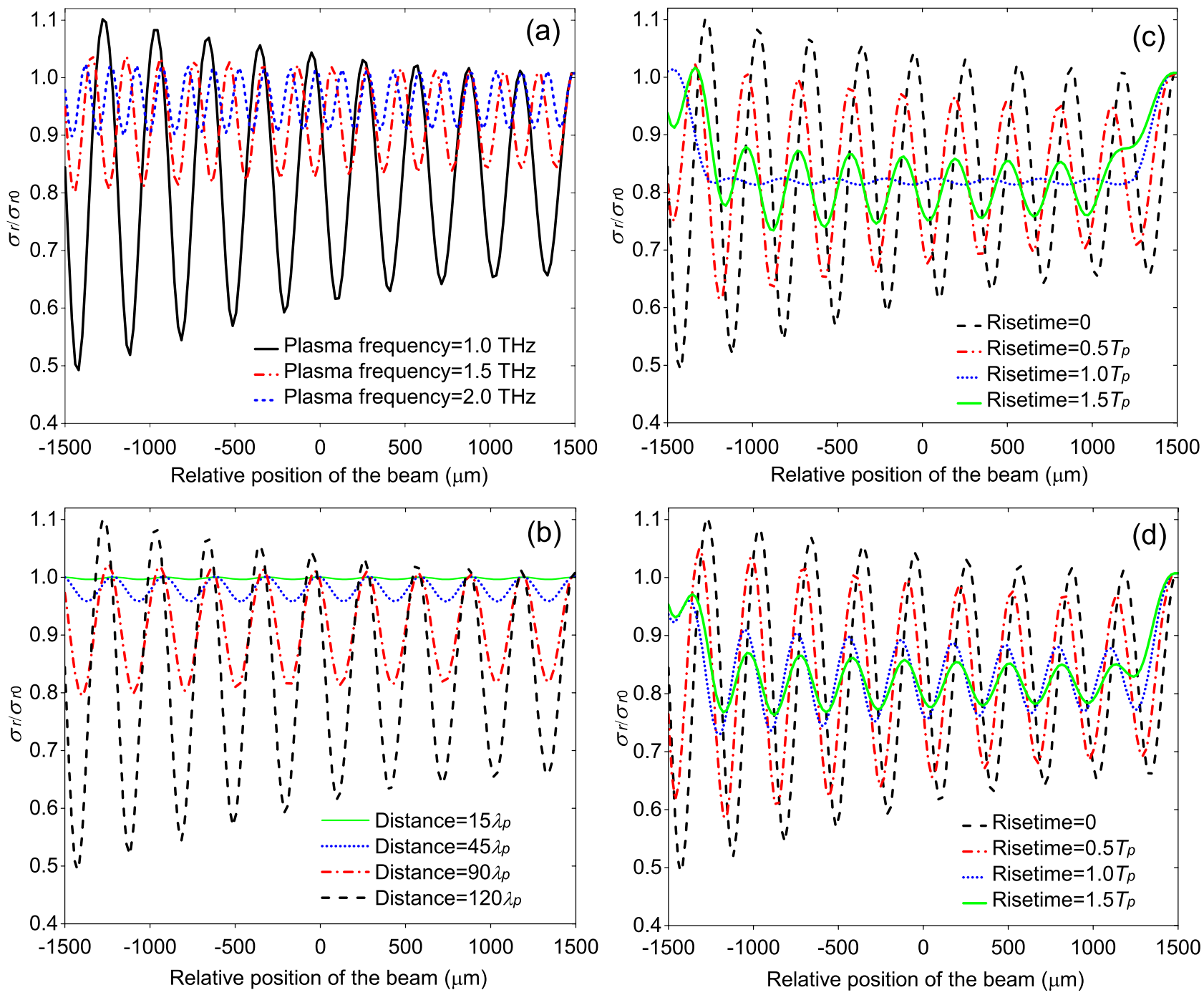

FIG. 1. The beam parameter $\sigma_{r} / \sigma_{r 0}$ plotted as a function of position along the plasma channel for: (a) different plasma frequencies $\left(L_{p}=3.6 \mathrm{~cm}\right)$; (b) different propagation distances $\left(\lambda_{p}=300 \mu \mathrm{m}\right) ;(\mathrm{c})$ various linear rise times $\left(T_{p}=1 \mathrm{ps}, L_{p}=120 \lambda_{p}\right) ;(\mathrm{d})$ various sinusoidal rise times $\left(T_{p}=1 \mathrm{ps}, L_{p}=120 \lambda_{p}\right)$. Other beam parameters are $E=50 \mathrm{MeV}$ and $Q=1 \mathrm{nC}$.

Here, it is used to investigate beam modulation in a plasma and the generation of coherent $\mathrm{THz}$ radiation in the near-field region. We start by modelling the beam modulation in a plasma channel. The simulations were carried out using a frame moving with the electron beam. In this case, we assume that the EM radiation outside the numerical box cannot affect the beam and the plasma dynamics inside the box. This is a reasonable approximation, taking into account that the beam travels with a speed close to the speed of light (ultra-relativistic beam) and allows a significant reduction of the calculation time. The radiation due to beam-plasma interactions in Refs. 30-33 is not considered in the beam modulation simulation process. The dimensions of the simulation box are $3300 \mu \mathrm{m}$ (longitudinal direction) by $600 \mu \mathrm{m}$ (transverse direction), and it is divided into 660 cells by 318 cells, respectively.

Figure 2 shows the modulated profile of a beam which has initial parameters as listed in Table I. The results are shown for beams with trapezoidal initial longitudinal profiles and various rise and decay times. Figures 2(b)-2(d) illustrate the results of the numerical studies and show the influence of the plasma density on a square beam. It can be seen that, after propagating a distance of $2.3 \mathrm{~cm}$, the beams have different periodicities and amplitudes of modulation. The beam in Fig. 2(b) is fully modulated with periodicity of $300 \mu \mathrm{m}$, while the beams in Figs. 2(c) and 2(d) are partially modulated with the respective periodicities of $200 \mu \mathrm{m}$ and $150 \mu \mathrm{m}$ confirming the results observed in Sec. II. It is worth noting that, for the same simulation parameters and the same propagation distance in the plasma, the modulation depth predicted by VSim is larger than that of the analytical model. The reasons for this are the subject of further investigations.

For Figs. 2(e)-2(h), the plasma density $n_{p}=1.24 \times 10^{16} /$ $\mathrm{cm}^{3}$ was fixed $\left(\lambda_{p}=300 \mu \mathrm{m}\right)$ and a trapezoidal bunch profile has been assumed. The bunch rise time has been varied as shown in the figure caption. Comparison of Fig. 2(b) with Figs. 2(e)-2(h) shows that the modulation rate is largest for a square beam. As observed before (Fig. 1), Fig. 2(e) shows that for a beam with a linear rise time equal to the plasma period, beam compression without periodical modulation is predicted. While for the same rise time, the modulation of the beam with a sinusoidal rise profile is clearly shown in Fig. 2(g). Additionally, Figs. 2(b)-2(d) show that the modulation for the trailing edge 


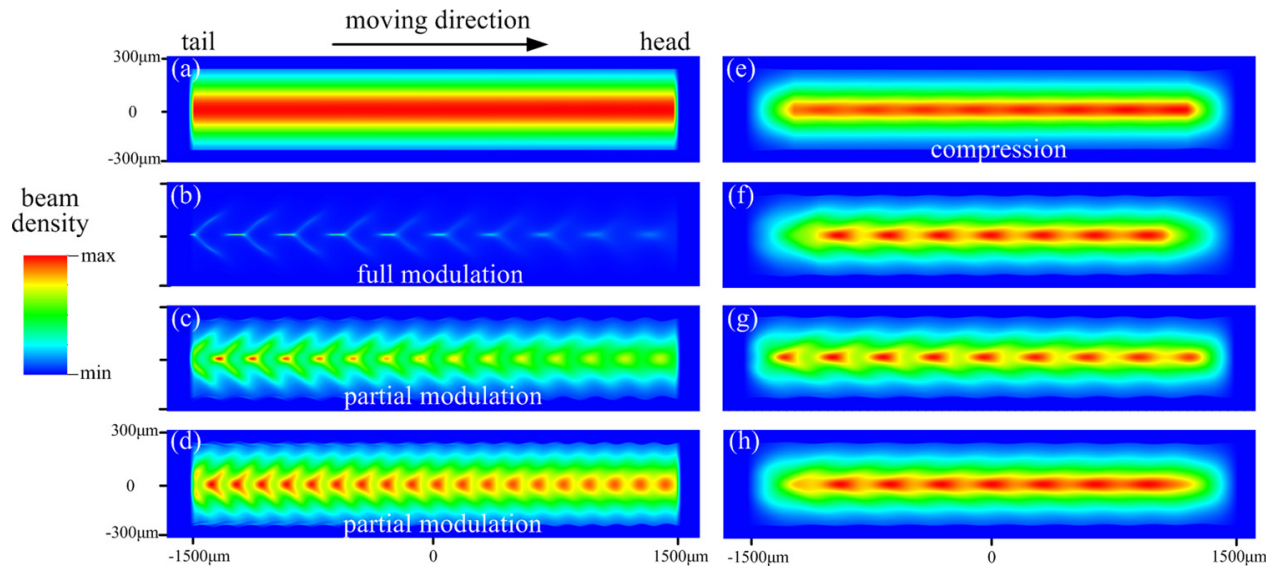

FIG. 2. Numerical simulations of the bunch modulation after propagating a distance of $2.3 \mathrm{~cm}$, for different plasma densities and beam profiles: (a) initial charge distribution with square profile; [(b)-(d)] square beam profile with a plasma wavelength of $300 \mu \mathrm{m}$ (b), $200 \mu \mathrm{m}$, (c) and $150 \mu \mathrm{m}$ (d), respectively; [(e)-(h)] plasma wavelength $\lambda_{p}=300 \mu \mathrm{m}\left(T_{p}=1 \mathrm{ps}\right)$, trapezoidal profile with linear rise time of $T_{p}$ (e) and $1.5 T_{p}$ (f), trapezoidal profile with sinusoidal rise time of $T_{p}(\mathrm{~g})$ and $1.5 T_{p}(\mathrm{~h})$. Other beam parameters are $E=50 \mathrm{MeV}$ and $Q=1 \mathrm{nC}$.

of the beam (left) is larger than that of the leading edge (right), which is in good agreement with the beam envelope model. The results observed in these sections underline the importance of initial beam profile conditioning, for example, profiling of the beam rise time to observe strong modulation within the shortest distance.

\section{THE PROPAGATION OF A MODULATED BEAM IN THE VACUUM AREA}

As discussed above, the beam modulation in plasma happens in the transverse plane due to the focusing and defocusing forces acting on the beam. This modulates the beam and makes its transverse charge distribution vary; nevertheless, the total current of the beam or total charge across a given cross section does not change. It means that there is no modulation of the beam current or of the number of electrons across each transverse plane, in spite of the fact that the transverse size of the defocused part of the beam is different from that of the focused part. In order to generate efficient $\mathrm{THz}$ radiation, one may try positioning the target close to the focused part of the beam but the necessity of avoiding the direct hit of the target by the defocused electrons will necessitate the positioning of the target well outside the beam trajectory. This would have a strong effect on the generation of EM radiation. To resolve this issue, one solution would be to remove the defocused part of the modulated beam, for example, by using a suitable collimator. The specific structure of the collimator is outside the scope of this paper.

The plasma modulated beam has to be transported to the target (a grating in our case) and separate numerical studies using VSim simulations have been done to investigate the transport of the beam from the plasma channel into vacuum and its propagation in the vacuum area under the effect of subsequent space-charge forces. First, the micro-bunched beam has to be brought from the plasma channel into the vacuum area by transferring all the beam parameters and selffields into a new simulation database. These parameters were then used as initial conditions at the start of the next stage. The schematic of the numerical box is shown in Fig. 3. The boundaries of the box were formed by a perfectly matched layer which absorbs the exiting EM waves and particles. The modulated beam (Fig. 2(b), modulation periodicity of $300 \mu \mathrm{m}$ ) was brought into the simulation area and collimated using an aperture with a diameter of $a=100 \mu \mathrm{m}$. After the collimator, the train of micro-bunches was only made up of radially focused electrons. The comparison of the longitudinal beam profile $n(z)$ before (solid black line) and after the collimator (red dashed line) is shown in Fig. 4. By removing the defocused part of the beam, a clear longitudinal modulation with a periodicity of 1 ps was observed.

In the vacuum area, the modulated beam experiences space-charge defocusing forces. Therefore, it is important to investigate if a micro-bunched beam can propagate to the $\mathrm{THz}$ target while maintaining the required periodicity and amplitude of modulation. In order to understand the stability of the beam modulation, beams of different energies have been considered. Using the beam parameters listed in Table I (by varying beam energy) and assuming that the plasma wavelength is $300 \mu \mathrm{m}$, the propagation of plasma modulated beams after collimation with energies of $10 \mathrm{MeV}, 20 \mathrm{MeV}$, and $50 \mathrm{MeV}$ was studied numerically. We note that higher beam energy leads to a smaller amplitude of beam density modulation (for a given length of the plasma channel). In order to get the same modulation for beams of different energies, plasma channels of

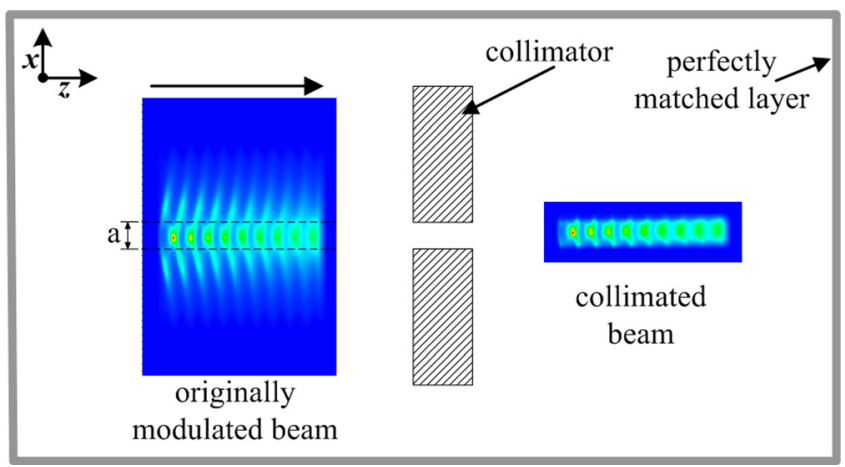

FIG. 3. A schematic of the VSim simulation showing the beam propagation through a collimator. 


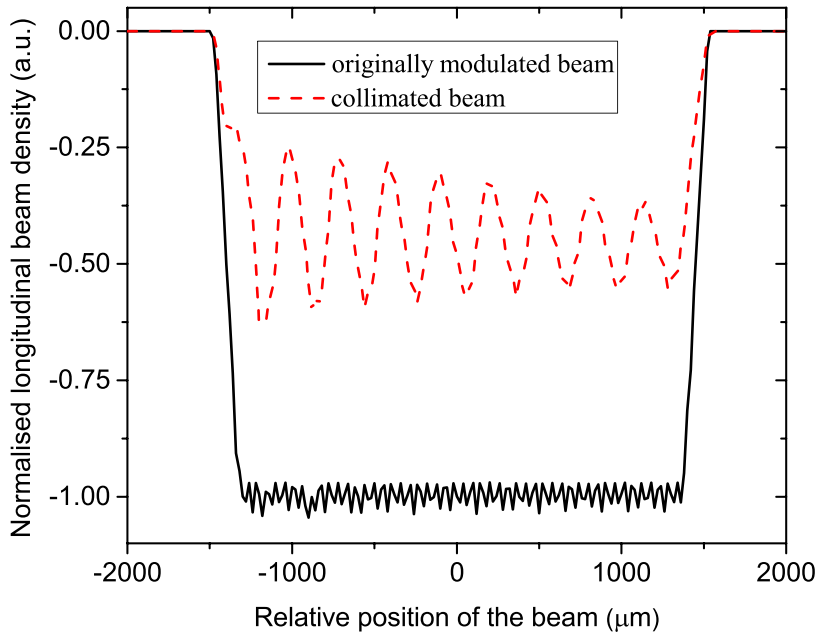

FIG. 4. The normalised longitudinal charge distribution along the beam propagation direction for the originally modulated beam and the collimated beam.

different lengths $1 \mathrm{~cm}(10 \mathrm{MeV}), 1.5 \mathrm{~cm}(20 \mathrm{MeV})$, and $2.2 \mathrm{~cm}$ $(50 \mathrm{MeV})$ were used in the simulations. The comparison of the collimated beam profiles after propagation over a distance of $1 \mathrm{~cm}$ (black dashed line) and $10 \mathrm{~cm}$ (solid red line) in the vacuum area is shown in Fig. 5. In this case, the longitudinal beam density is the integration of all the particles within a $200 \mu \mathrm{m}$ diameter around the beam axis.

For beams with energies above $20 \mathrm{MeV}$, the longitudinal modulation of the micro-bunched beam can be preserved over longer distances than $10 \mathrm{~cm}$ used in the simulation. However, the preservation of the beam modulation for energies below $20 \mathrm{MeV}$ is not as good. One of the possible solutions, for beam energies below $20 \mathrm{MeV}$, would be to reduce the micro-bunch charge by increasing the overall train length while maintaining the total charge. This would avoid beam dispersion due to the space-charge effect of individual micro-bunches. With decreasing energy, the number of electrons reaching the end of the simulation range becomes smaller due to transverse dispersion, as can be seen from comparison of Figs. 5(b) and 5(f). In practice, an increase in the beam energy would also increase the distance over which modulation can be maintained. This would be an advantage for any practical application.

\section{THE GENERATION OF TUNABLE THz RADIATION IN THE NEAR-FIELD REGION}

The $\mathrm{THz}$ radiation generation from the micro-bunched beams has been investigated using VSim. In general, SmithPurcell, Cherenkov, transition or diffraction radiation mechanisms can be explored for $\mathrm{THz}$ radiation generation but only the Smith-Purcell mechanism will be considered in this work. Smith-Purcell radiation (SPr) is observed when an electron beam propagates above a periodic metallic grating. The motion of the induced charges on the surface of the grating by the beam will generate the radiation. ${ }^{10,34-37}$ When the transverse and longitudinal dimensions of a single micro-bunch are smaller than the operating wavelength, the radiation generated by the bunch is coherent. ${ }^{4}$ Assuming that the transverse dimensions of the beam are much smaller than the transverse dimensions of the grating, one can use a $2 \mathrm{D}$ approximation for the numerical model. Field distribution along the transverse direction is not discussed here due to this restriction on the model. As far-field region calculations of this kind require excessive amounts of computing resources, our simulations were carried out in the near-field region.

The schematic of the model is shown in Fig. 6 with the grating located below the beam. The same boundaries as shown in Fig. 3 were used to define the numerical box. The
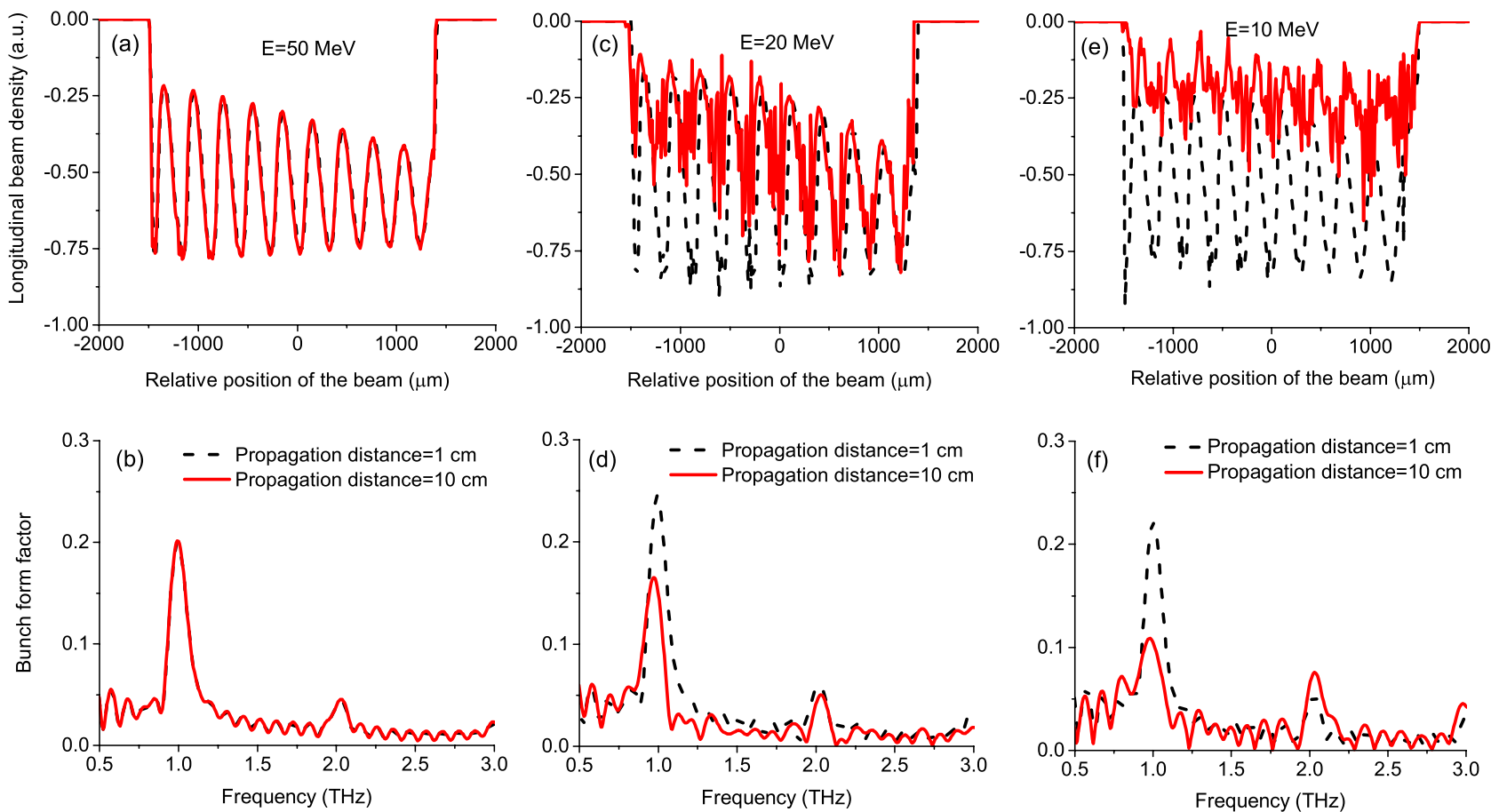

FIG. 5. Comparison of the beam profiles (top) and the corresponding form factors (bottom) after propagating $1 \mathrm{~cm}$ (black dashed line) and $10 \mathrm{~cm}$ (solid red line) in the vacuum area when the beam energy is: $50 \mathrm{MeV}$ [(a) and (b)]: $20 \mathrm{MeV}$ (c, d); $10 \mathrm{MeV}$ [(e) and (f)]. 


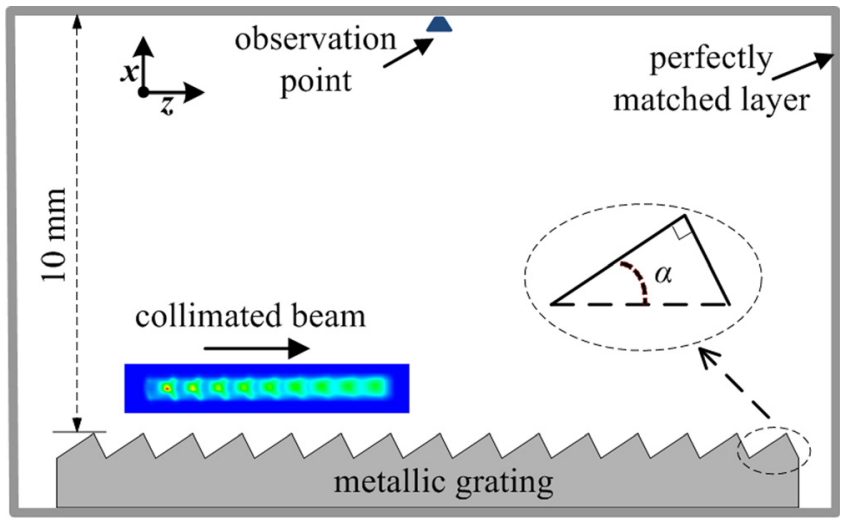

FIG. 6. A schematic of the VSim simulation showing $\mathrm{THz}$ radiation generation.

grating was assumed to have a length of $12.5 \mathrm{~mm}$, a periodicity of $0.5 \mathrm{~mm}$, and a facet blaze angle $\alpha=30^{\circ}$. The distance between the beam centre and the grating was $0.5 \mathrm{~mm}$. The field monitor, used to record the magnetic field in the $y$ direction $\left(B_{y}\right)$ in the near-field region, was positioned $10 \mathrm{~mm}$ above the grating centre and at $90^{\circ}$ to its surface.

By changing the periodicity of the modulated beams, it is possible to observe the tunability of the emitted $\mathrm{THz}$ radiation. Figure 7 shows the pre-bunched beams, with microbunching spacings of $300 \mu \mathrm{m}, 200 \mu \mathrm{m}$, and $150 \mu \mathrm{m}$ that were used in the numerical simulations. The energy loss of the highly relativistic beams due to their interaction with the periodic metal grating is negligible. The bunch profiles of the prebunched beams are kept constant, while they propagate above the grating. The Fourier transforms of radiation signals $B_{y}$ generated by these micro-bunch trains are shown in Fig. 8.

Traditionally, SPr is investigated in the far-field region, which means that the dispersion relation which relates the radiated frequency to the emission angle is always fulfilled. The dispersion relation is as follows:

$$
\lambda=\frac{l}{n}\left(\frac{1}{\beta}-\cos \theta\right)
$$

where $\lambda$ is the radiation wavelength, $l$ is the periodicity of the grating, $n$ is the order of the radiation, $\beta=v / c$ is the

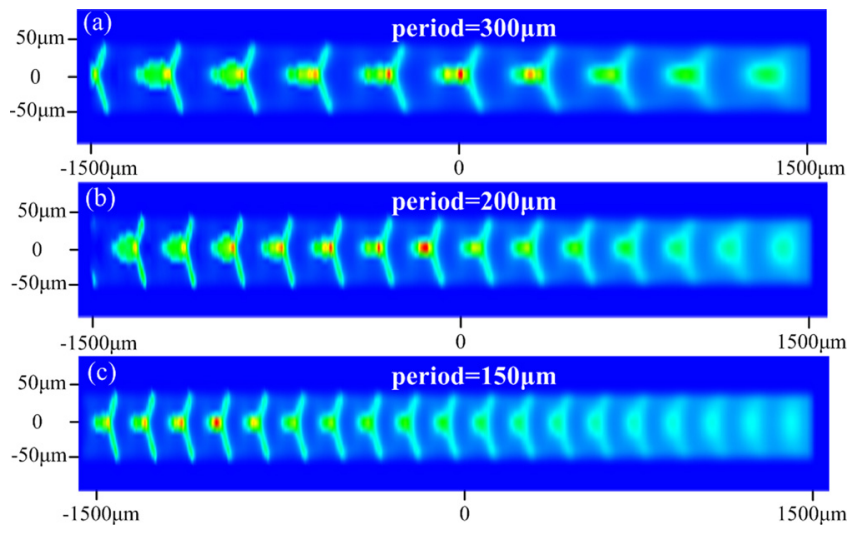

FIG. 7. Contour plots of the charge distribution for the micro-bunch trains after beam collimation with different micro-bunching spacing: $300 \mu \mathrm{m}$ (a), $200 \mu \mathrm{m}$ (b), and $150 \mu \mathrm{m}$ (c). Beam energy $=50 \mathrm{MeV}$.
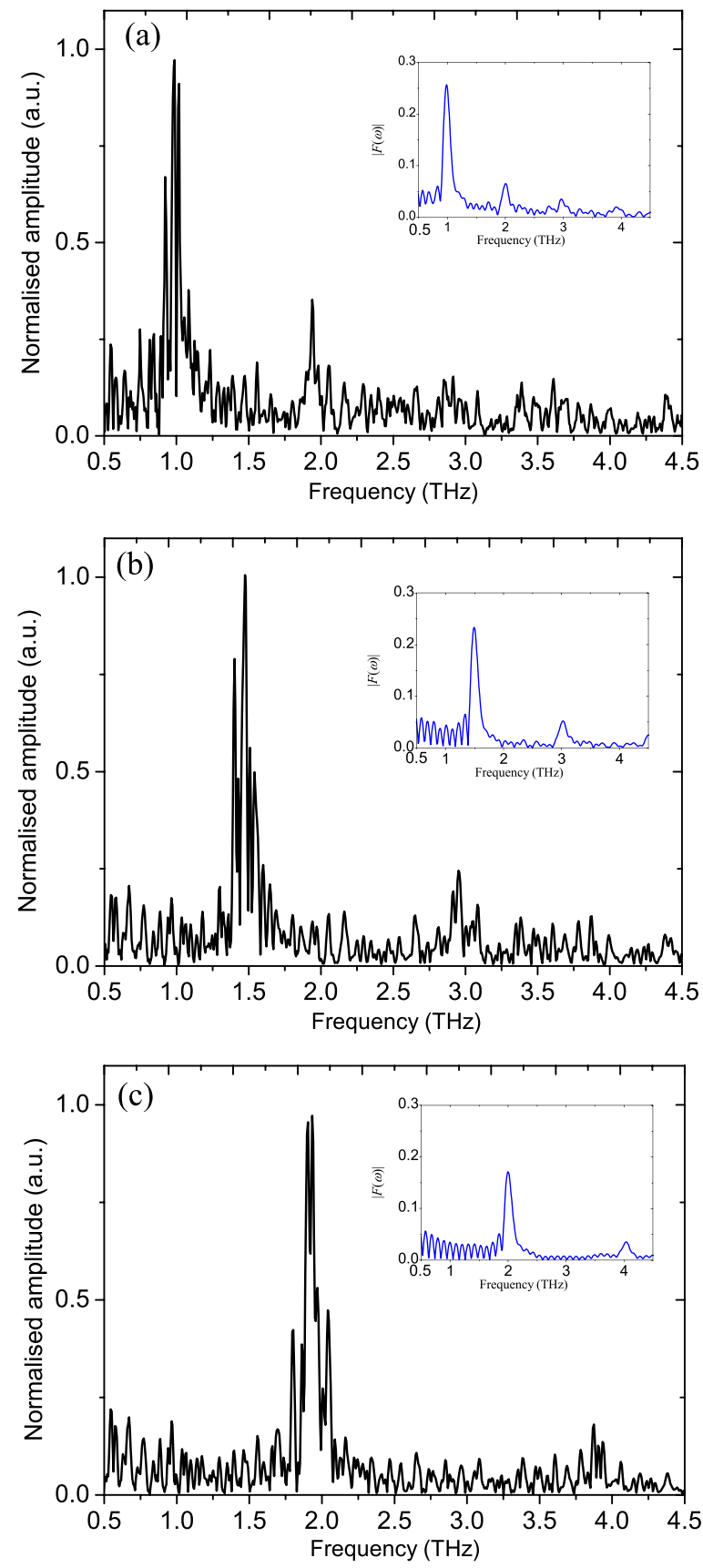

FIG. 8. Normalised amplitude of the emitted radiation from the microbunch trains after beam collimation (insets are the form factor of the corresponding modulated beams in Fig. 7).

relativistic factor of the beam, and $\theta$ is the observation angle relative to the beam propagation direction.

In Fig. 8, however, there is no monochromatic radiation at a frequency of $0.6 \mathrm{THz}$ (grating period of $0.5 \mathrm{~mm}$ at observation angle $90^{\circ}$ ). In this case, the monitor collects the whole spectrum and without the interference effects, the dispersion relation is not satisfied. The existence of the micro-bunch trains ensures that the spectrum in the near-field region is dominated by the component which corresponds to the bunch spacing not the spectrum of a single bunch. Our simulations have also demonstrated that spectra as shown in Fig. 8 are unaffected by small changes in the beam-grating separation or in the position of the monitor point used in the simulations. 
The frequency of radiation is determined by the microbunch spacing and can be changed by adjusting the bunch train periodicity. The tunability of the radiation is achieved by varying the periodicity of the micro-bunch train which in turn can be controlled by the plasma density. In this case, a change of the grating period is not required. Assuming plasma density in the range of $10^{15} / \mathrm{cm}^{3}$ to $10^{17} / \mathrm{cm}^{3}$, the tunable frequency range would be between $0.3 \mathrm{THz}$ and $3 \mathrm{THz}$. This proposed system could potentially be less complex and costly than a $\mathrm{THz}$ Free Electron Laser for the same frequency ranges.

\section{CONCLUSION}

We have discussed the modulation of a picoseconds long relativistic electron beam into a train of micro-bunches. The interaction between the long electron bunch and a plasma channel was studied using both analytical theory and numerical simulations. Based on the analytical beam envelope model, the parameters which affect the beam modulation have been analysed, including the plasma density, the beam propagation distance inside the plasma, and the longitudinal profile of the original, unmodulated bunch. Good agreement between PIC simulations and the analytical model was observed. The beam collimation required to observe efficient transfer of the beam energy into $\mathrm{THz}$ radiation was also studied. Our simulations show that the interaction of a plasma modulated and collimated beam with a metallic grating would result in the emission of coherent $\mathrm{THz}$ radiation with a spectrum determined by the micro-bunch spacing in the near-field region. Hence, it would be possible to consider a tunable $\mathrm{THz}$ radiation source where the tunability is achieved by changing the bunch spacing, which is in turn controlled by the plasma density. We believe that this approach has the potential to help bridge the "THz gap" through the development of a versatile, compact, affordable, and tunable source of coherent $\mathrm{THz}$ radiation.

\section{ACKNOWLEDGMENTS}

The authors would like to acknowledge the partial support of the project from the STFC UK through PRD Grant No. ST/M003590/1, the Leverhulme Trust through the International Network Grant No. IN-2015-012. The authors would also like to thank $\mathrm{H}$. Harrison for helping with editing the text.

\section{APPENDIX A: THE BEAM INTERACTION MODEL}

For a beam moving through a plasma channel while having charge density much less than the electron density of the plasma, the plasma wakefield generated can be described analytically using linear wakefield theory. ${ }^{25,26}$ Here, we consider only the radial forces and their effect on beam dynamics. To describe this, the slow amplitude envelope equation for the azimuthally symmetric beam can be written as ${ }^{27}$

$$
\frac{\partial^{2} \sigma_{r}}{\partial z^{2}}-\frac{\varepsilon^{2}}{\sigma_{r}^{3}}-\frac{1}{\gamma m_{0} c^{2}} \frac{\left\langle r W_{r}\right\rangle}{2 \sigma_{r}}=0,
$$

where $\sigma_{r}=\sqrt{\left\langle r^{2} / 2\right\rangle}$ is the beam transverse size, $\varepsilon$ is the beam r.m.s emittance, $\gamma$ is the beam relativistic factor, $W_{r}$ is the radial force acting on a single electron, and $z$ is the longitudinal coordinate along the beam propagation. We note that no acceleration was assumed due to longitudinal wakefields $\left(\frac{\partial \gamma}{\partial z}=0\right)$, in other words no beam modulation due to the longitudinal velocity modulation. Also the beam hosing instability is ignored here, $(\langle r\rangle=0)$. In the axisymmetric cylindrical system, $\left\langle r^{2}\right\rangle=\int_{0}^{+\infty} r^{2} n_{b}(r) r d r / \int_{0}^{+\infty} n_{b}(r) r d r$, which is the average of all electrons in the transverse direction. The radial force $W_{r}$ can be written as

$$
\begin{aligned}
W_{r}(\xi, r, z) & =W_{r 1}(\xi, r, z)+W_{r 2}(\xi, r, z), \\
W_{r 1}(\xi, r, z) & =-\frac{e^{2}}{\epsilon_{0} k_{p e}} \int_{+\infty}^{\xi} \sin k_{p e}\left(\xi-\xi^{\prime}\right) R\left(\xi^{\prime}, r, z\right) d \xi^{\prime}, \\
W_{r 2}(\xi, r, z) & =\frac{e^{2}}{\epsilon_{0} k_{p e}^{2} \gamma^{2}} R(\xi, r, z),
\end{aligned}
$$

with

$$
\begin{aligned}
R(\xi, r, z)= & -k_{p e}^{3} K_{1}\left(k_{p e} r\right) \int_{0}^{r} r^{\prime} d r^{\prime} n_{b}\left(\xi, r^{\prime}, z\right) I_{0}\left(k_{p e} r^{\prime}\right) \\
& +k_{p e}^{3} I_{1}\left(k_{p e} r\right) \int_{r}^{+\infty} r^{\prime} d r^{\prime} n_{b}\left(\xi, r^{\prime}, z\right) K_{0}\left(k_{p e} r^{\prime}\right),
\end{aligned}
$$

where $W_{r 1}$ is the focusing force caused by the plasma perturbation, $W_{r 2}$ represents the defocusing force due to the electrons self-repulsion within the beam, $R(\xi, r, z)$ describes the radial bunch profile dependence of the wakefield, $k_{p e}$ is the plasma wave number, $\xi=-v_{b} t+z$ is a co-moving variable, which means the relative distance from the middle of the beam, $n_{b}(\xi, r, z)$ is beam charge density distribution, and $I_{0,1}$ and $K_{0,1}$ are the modified Bessel functions of the zeroth and first order.

\section{APPENDIX B: TRANSVERSE WAKEFIELD ANALYSIS}

The analysis of the dependence of the modulation of the initial long bunch on its properties, and specifically on its rise time and on the shape of the leading edges, is as follows: assuming that the initial particle distributions in the longitudinal and transverse directions are uncorrelated, the beam density can be written as

$$
n_{b}(\xi, r)=n_{b_{\|}}(\xi) n_{b_{\perp}}(r) .
$$

Thus, the transverse force $W_{r 1}$ can be written as $W_{r 1}$ $=f(\xi) g(r)$, and we have

$$
\begin{aligned}
f(\xi)= & -\frac{e^{2}}{\epsilon_{0} k_{p e}} \int_{+\infty}^{\xi} n_{b_{\|}}\left(\xi^{\prime}\right) \sin k_{p e}\left(\xi-\xi^{\prime}\right) d \xi^{\prime}, \\
g(r)= & -k_{p e}^{3} K_{1}\left(k_{p e} r\right) \int_{0}^{r} r^{\prime} d r^{\prime} n_{b_{\perp}}\left(r^{\prime}\right) I_{0}\left(k_{p e} r^{\prime}\right) \\
& +k_{p e}^{3} I_{1}\left(k_{p e} r\right) \int_{r}^{+\infty} r^{\prime} d r^{\prime} n_{b_{\perp}}\left(r^{\prime}\right) K_{0}\left(k_{p e} r^{\prime}\right),
\end{aligned}
$$

where $f(\xi)$ depends on the longitudinal profile $n_{b_{\|}}(\xi)$, and $g(r)$ depends on the transverse profile $n_{b_{\perp}}(r)$. 


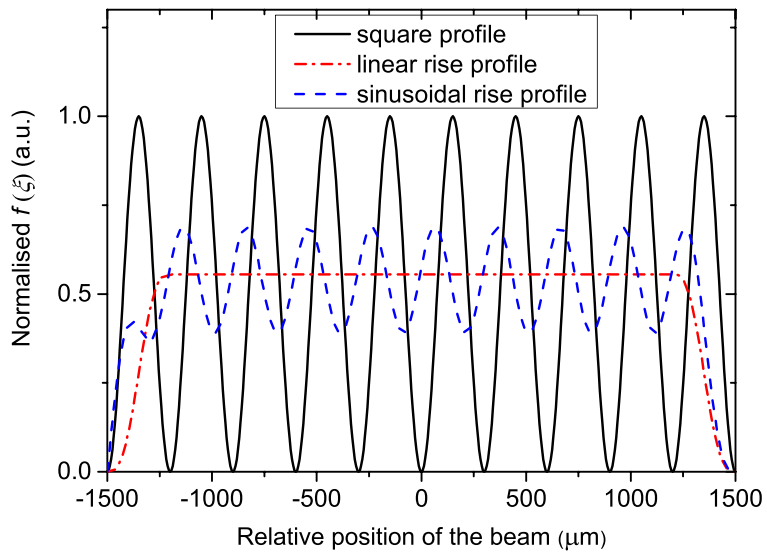

FIG. 9. Plot of $f(\xi)$ as a function of the relative beam position for different bunch profiles (plasma period $T_{p}=1 \mathrm{ps}$ ): square profile (solid black line); trapezoidal profile with linear rise time of $T_{p}$ (red dashed-dotted line); trapezoidal profile with sinusoidal rise time of $T_{p}$ (blue dashed line).

The dependence of $f(\xi)$ on the longitudinal profile is shown in Fig. 9. For the profile with linear rise time equal to the plasma period, there is no oscillation of the wakefield component $f(\xi)$, while the oscillating component does not become zero for sinusoidal rise beam or square beam. Therefore, in the case of the beam with linear rise time equal to a plasma period there is only spatially constant force acting on the beam and the beam is not modulated, while a beam with sinusoidal rise time also equal to a plasma period can still be modulated.

${ }^{1}$ E. Brundermann, H.-W. Hubers, and M. F. Kimmitt, Terahertz Techniques (Springer, 2012).

${ }^{2}$ M. Tonouchi, Nat. Photonics 1, 97 (2007).

${ }^{3}$ S. Dhillon, M. Vitiello, E. Linfield, A. Davies, M. C. Hoffmann, J. Booske, C. Paoloni, M. Gensch, P. Weightman, G. Williams et al., J. Phys. D: Appl. Phys. 50, 043001 (2017).

${ }^{4}$ G. L. Carr, M. C. Martin, W. R. McKinney, K. Jordan, G. R. Neil, and G. P. Williams, Nature 420, 153 (2002).

${ }^{5}$ A. Cook, R. Tikhoplav, S. Y. Tochitsky, G. Travish, O. Williams, and J. Rosenzweig, Phys. Rev. Lett. 103, 095003 (2009).

${ }^{6}$ G. Andonian, O. Williams, X. Wei, P. Niknejadi, E. Hemsing, J. Rosenzweig, P. Muggli, M. Babzien, M. Fedurin, K. Kusche et al., Appl. Phys. Lett. 98, 202901 (2011).

${ }^{7}$ S. Antipov, M. Babzien, C. Jing, M. Fedurin, W. Gai, A. Kanareykin, K. Kusche, V. Yakimenko, and A. Zholents, Phys. Rev. Lett. 111, 134802 (2013).

${ }^{8}$ Z. Zhang, L. Yan, Y. Du, Z. Zhou, X. Su, L. Zheng, D. Wang, Q. Tian, W. Wang, J. Shi et al., Phys. Rev. Lett. 116, 184801 (2016).

${ }^{9}$ J. H. Booske, R. J. Dobbs, C. D. Joye, C. L. Kory, G. R. Neil, G.-S. Park, J. Park, and R. J. Temkin, IEEE Trans. Terahertz Sci. Technol. 1, 54 (2011).
${ }^{10}$ G. Doucas, V. Blackmore, B. Ottewell, C. Perry, P. Huggard, E. CastroCamus, M. Johnston, J. L. Hughes, M. Kimmitt, B. Redlich et al., Phys. Rev. Spec. Top.-Accel. Beams 9, 092801 (2006).

${ }^{11}$ H. L. Andrews, F. Bakkali Taheri, J. Barros, R. Bartolini, V. Bharadwaj, C. Clarke, N. Delerue, G. Doucas, N. Fuster-Martinez, M. VieilleGrosjean, I. V. Konoplev, M. Labat, S. Le Corre, C. Perry, A. Reichold, and S. Stevenson, Phys. Rev. Spec. Top. -Accel. Beams 17, 052802 (2014).

${ }^{12}$ S. I. Bajlekov, M. Heigoldt, A. Popp, J. Wenz, K. Khrennikov, S. Karsch, and S. M. Hooker, Phys. Rev. Spec. Top. -Accel. Beams 16, 040701 (2013).

${ }^{13}$ T. J. Maxwell, C. Behrens, Y. Ding, A. S. Fisher, J. Frisch, Z. Huang, and H. Loos, Phys. Rev. Lett. 111, 184801 (2013).

${ }^{14}$ Y. Shen, X. Yang, G. Carr, Y. Hidaka, J. B. Murphy, and X. Wang, Phys. Rev. Lett. 107, 204801 (2011).

${ }^{15}$ P. Musumeci, R. Li, and A. Marinelli, Phys. Rev. Lett. 106, 184801 (2011).

${ }^{16}$ J. Neumann, R. Fiorito, P. O'Shea, H. Loos, B. Sheehy, Y. Shen, and Z. Wu, J. Appl. Phys. 105, 053304 (2009).

${ }^{17}$ S. Bielawski, C. Evain, T. Hara, M. Hosaka, M. Katoh, S. Kimura, A. Mochihashi, M. Shimada, C. Szwaj, T. Takahashi et al., Nat. Phys. 4, 390 (2008).

${ }^{18}$ Z. Zhang, L. Yan, Y. Du, W. Huang, C. Tang, and Z. Huang, Phys. Rev. Accel. Beams 20, 050701 (2017).

${ }^{19}$ S. Antipov, C. Jing, M. Fedurin, W. Gai, A. Kanareykin, K. Kusche, P. Schoessow, V. Yakimenko, and A. Zholents, Phys. Rev. Lett. 108, 144801 (2012).

${ }^{20}$ P. Muggli, V. Yakimenko, M. Babzien, E. Kallos, and K. Kusche, Phys. Rev. Lett. 101, 054801 (2008).

${ }^{21}$ P. Muggli, B. Allen, V. Yakimenko, J. Park, M. Babzien, K. Kusche, and W. Kimura, Phys. Rev. Spec. Top.-Accel. Beams 13, 052803 (2010).

${ }^{22}$ Y.-E. Sun, P. Piot, A. Johnson, A. Lumpkin, T. Maxwell, J. Ruan, and R. Thurman-Keup, Phys. Rev. Lett. 105, 234801 (2010).

${ }^{23}$ N. Kumar, A. Pukhov, and K. Lotov, Phys. Rev. Lett. 104, 255003 (2010).

${ }^{24}$ Y. Fang, V. Yakimenko, M. Babzien, M. Fedurin, K. Kusche, R. Malone, J. Vieira, W. Mori, and P. Muggli, Phys. Rev. Lett. 112, 045001 (2014).

${ }^{25}$ T. Katsouleas, S. Wilks, P. Chen, J. Dawson, and J. Su, Part. Accel. 22, 81 (1987).

${ }^{26}$ Y. Fang, J. Vieira, L. Amorim, W. Mori, and P. Muggli, Phys. Plasmas 21, 056703 (2014)

${ }^{27}$ R. Govil, W. Leemans, E. Y. Backhaus, and J. Wurtele, Phys. Rev. Lett. 83, 3202 (1999).

${ }^{28}$ J. Vieira, L. Amorim, Y. Fang, W. Mori, P. Muggli, and L. Silva, Plasma Phys. Controlled Fusion 56, 084014 (2014).

${ }^{29}$ C. Nieter and J. R. Cary, J. Comput. Phys. 196, 448 (2004).

${ }^{30}$ I. Timofeev, V. Annenkov, and A. Arzhannikov, Phys. Plasmas 22, 113109 (2015)

${ }^{31}$ V. Annenkov, I. Timofeev, and E. Volchok, Phys. Plasmas 23, 053101 (2016).

${ }^{32}$ I. Timofeev, E. Volchok, and V. Annenkov, Phys. Plasmas 23, 083119 (2016).

${ }^{33}$ I. V. Timofeev, E. A. Berendeev, and G. I. Dudnikova, Phys. Plasmas 24, 093114 (2017)

${ }^{34}$ S. J. Smith and E. Purcell, Phys. Rev. 92, 1069 (1953).

${ }^{35}$ F. Rusin and G. Bogomolov, Proc. IEEE 57, 720 (1969).

${ }^{36}$ I. Konoplev, A. MacLachlan, C. Robertson, A. Cross, and A. Phelps, Appl. Phys. Lett. 101, 121111 (2012).

${ }^{37}$ N. Glass, Phys. Rev. A 36, 5235 (1987). 